\title{
Quantization of Damped Systems Using Fractional WKB Approximation
}

\author{
Ola A. Jarab'ah ${ }^{1}$ \\ ${ }^{1}$ Applied Physics Department, Faculty of Science, Tafila Technical University, Tafila, Jordan \\ Correspondence: Ola A. Jarab'ah. Applied Physics Department, Faculty of Science, Tafila Technical University, \\ Tafila, Jordan. E-mail: oasj85@yahoo.com
}

Received: July 25, 2018

Accepted: August 12, 2018

Online Published: September 27, 2018

doi:10.5539/apr.v10n5p34

URL: https://doi.org/10.5539/apr.v10n5p34

\begin{abstract}
The Hamilton Jacobi theory is used to obtain the fractional Hamilton-Jacobi function for fractional damped systems. The technique of separation of variables is applied here to solve the Hamilton Jacobi partial differential equation for fractional damped systems. The fractional Hamilton-Jacobi function is used to construct the wave function and then to quantize these systems using fractional WKB approximation. The solution of the illustrative example is found to be in exact agreement with the usual classical mechanics for regular Lagrangian when fractional derivatives are replaced with the integer order derivatives and $\gamma \rightarrow 0$.
\end{abstract}

Keywords: Damped Systems, WKB Approximation, Wave Function, Hamilton-Jacobi Function, Hamilton Jacobi Equation

\section{Introduction}

The Hamilton Jacobi theory provides a bridge between classical and quantum mechanics; it implies that quantum mechanics should reduce to classical mechanics in the limit $\hbar \rightarrow 0$. The principal interest in this theory is based on the hope that it might provide some guidance concerning the form of a Schrödinger type quantum theory for constrained fields. The fact that (Arnold, 1989; Goldstein, 1980; Lanczos, 1986) solving the Hamilton Jacobi equation gives a generating function for the family of canonical transformation of the dynamics is the theoretical basis for the powerful technique of exact integration of Hamilton's equations that are often employed with the technique of separation of variables.

The canonical formalism for investigating the first order singular systems has been developed by (Guler, 1992; Rabei et al., 1992, Nawafleh, 1998; Rabei, 1999; Muslih, 2002). The quantization of constrained systems has been studied using the WKB approximation (Rabei et al., 2002, 2005; Hasan et al., 2004). The set of Hamilton Jacobi partial differential equations for these systems has been determined using the canonical method, the Hamilton Jacobi function has been obtained by solving these equations. In addition (Nawafleh et al., 2004; Nawafleh, 2007) calculating the Hamilton Jacobi function enables us to construct the wave function of constrained systems, for which the constraints become conditions on it in the semiclassical limit.

This limit also is known as the WKB approximation and it is named after physicists Wentzel, Kramers and Brillouin who all developed it in 1962.

Recently, the Hamilton Jacobi partial differential equations and WKB approximation have been studied for systems containing fractional derivatives using the canonical method (Rabei et al., 2009, 2010). More recently, a powerful approach, the canonical method, has been developed for dissipative systems (Jarab'ah et al., 2013). In this approach the equations of motion are written as total differential equations and the formulation leads to a set of Hamilton Jacobi partial differential equations which are familiar to regular systems. The purpose of this work is indeed to quantize the damped systems using fractional WKB approximation building on the previous work (Hasan, 2016).

This paper is organized as follows: In section 2, Hamilton Jacobi formalism and fractional WKB approximation are discussed. In section $\mathbf{3}$, one illustrative example is studied in detail. The work closes with some concluding remarks in section 4 . 


\section{Hamilton-Jacobi Formulation and Fractional WKB Approximation}

The Lagrangian formulation for time independent damped systems depending on the fractional derivatives is given by

$$
L\left(q,{ }_{a} D_{t}^{\alpha} q,{ }_{t} D_{b}^{\beta} q\right)=L_{\circ}\left(q,{ }_{a} D_{t}^{\alpha} q,{ }_{t} D_{b}^{\beta} q\right) e^{\gamma q}
$$

$e^{\gamma q}$ : time independent damping factor.

The formulation of fractional Euler Lagrange equation is obtained as

$$
\frac{\partial L}{\partial q}+{ }_{t} D_{b}^{\alpha} \frac{\partial L}{\partial_{a} D_{t}^{\alpha} q}+{ }_{a} D_{t}^{\beta} \frac{\partial L}{\partial_{t} D_{b}^{\beta} q}=0
$$

Remembering that:

The left Riemann- Liouville fractional derivative is defined as

$$
{ }_{a} D_{x}^{\alpha} f(x)=\frac{1}{\Gamma(n-\alpha)}\left(\frac{d}{d x}\right)^{n} \int_{a}^{x}(x-\tau)^{n-\alpha-1} f(\tau) d \tau
$$

which is denoted as the LRLFD,

and the right Riemann- Liouville fractional derivative is defined as

$$
{ }_{x} D_{b}^{\alpha} f(x)=\frac{1}{\Gamma(n-\alpha)}\left(-\frac{d}{d x}\right)^{n} \int_{x}^{b}(\tau-x)^{n-\alpha-1} f(\tau) d \tau
$$

which is denoted as the RRLFD,

If $\alpha$ is an integer, these derivatives are defined as follows:

$$
\begin{aligned}
{ }_{a} D_{x}^{\alpha} f(x) & =\left(\frac{d}{d x}\right)^{\alpha} f(x) \\
{ }_{x} D_{b}^{\alpha} f(x) & =\left(-\frac{d}{d x}\right)^{\alpha} f(x) \\
\alpha & =1,2, \ldots
\end{aligned}
$$

And the fractional Hamiltonian of damped systems is given by

$$
H\left(q, p_{\alpha}, p_{\beta}\right)=p_{\alpha a} D_{t}^{\alpha} q+p_{\beta t} D_{b}^{\beta} q-L\left(q,{ }_{a} D_{t}^{\alpha} q,{ }_{t} D_{b}^{\beta} q\right)
$$

The conjugate momenta can be written as

$$
\begin{gathered}
p_{\alpha}=\frac{\partial L}{\partial_{a} D_{t}^{\alpha} q} \\
p_{\beta}=\frac{\partial L}{\partial_{t} D_{b}^{\beta} q} \\
D_{t}^{1}=\frac{d}{d t}
\end{gathered}
$$

Following to the canonical method (Rabei et al., 1992) the Hamilton Jacobi partial differential equation reads as

$$
H^{\prime}=p_{\circ}+H
$$

Remembering that

$$
p_{\circ}=\frac{\partial S}{\partial t}
$$

Where $\mathbf{S}$ is the Hamilton Jacobi function and takes this form 


$$
S=S\left({ }_{a} D_{t}^{\alpha-1} q,{ }_{t} D_{b}^{\beta-1} q, E_{1}, E_{2}, t\right)
$$

so that equations $(11,12)$ can be written in compact form as

$$
H^{\prime}=\frac{\partial S}{\partial t}+H=0
$$

The solution of the above Hamilton Jacobi partial differential equation can be constructed as

$$
S=S\left({ }_{a} D_{t}^{\alpha-1} q,{ }_{t} D_{b}^{\beta-1} q, E_{1}, E_{2}, t\right)=f(t)+W_{1}\left(E_{1},{ }_{a} D_{t}^{\alpha-1} q\right)+W_{2}\left(E_{2},{ }_{t} D_{b}^{\beta-1} q\right)+A
$$

Where $E_{1}$ and $E_{2}$ are the constants of integration and $\mathbf{A}$ is some other constant. Thus, the equations of motion can be obtained using the canonical transformations as follows

$$
\begin{gathered}
\eta_{1}=\frac{\partial S}{\partial E_{1}}={ }_{a} D_{t}^{\alpha-1} Q \\
\eta_{2}=\frac{\partial S}{\partial E_{2}}={ }_{t} D_{b}^{\beta-1} Q \\
p_{\alpha}=\frac{\partial S}{\partial_{a} D_{t}^{\alpha-1} q}=\frac{\partial W_{1}}{\partial_{a} D_{t}^{\alpha-1} q} \\
p_{\beta}=\frac{\partial S}{\partial_{t} D_{b}^{\beta-1} q}=\frac{\partial W_{2}}{\partial_{t} D_{b}^{\beta-1} q}
\end{gathered}
$$

Where $\eta_{1}$ and $\eta_{2}$ are constants and can be determined from the initial conditions

The semiclassical expansion (WKB approximation) of Hamilton Jacobi function of constrained systems has been investigated by (Rabei et al., 2002). Following this reference the wave function can be constructed as

$$
\psi(q, t)=\prod_{i=1}^{N} \psi_{0 i}\left(q_{i}\right) \exp \left[\frac{i}{\hbar} S(q, t)\right]
$$

where $\psi_{0 i}\left(q_{i}\right)$ is the amplitude of the wave function, which is defined as

$$
\psi_{0 i}\left(q_{i}\right)=\frac{1}{\sqrt{p_{i}}}
$$

The wave function $\psi(q, t)$ satisfies the condition

$$
\hat{H}^{\prime} \psi=0
$$

In the semiclassical limits $\hbar \rightarrow 0$.

Thus, the wave function for damped systems in the fractional form can be written as

$$
\psi\left({ }_{a} D_{t}^{\alpha-1} q,{ }_{t} D_{b}^{\beta-1} q, t\right)=\frac{1}{\sqrt{p_{\alpha} p_{\beta}}} \exp \left[\frac{i}{\hbar} S\left({ }_{a} D_{t}^{\alpha-1} q,{ }_{t} D_{b}^{\beta-1} q, E_{1}, E_{2}, t\right)\right]
$$

And the momenta operators

$$
\begin{gathered}
\hat{p}_{\alpha}=\frac{\hbar}{i} \frac{\partial}{\partial_{a} D_{t}^{\alpha-1} q} \\
\hat{p}_{\beta}=\frac{\hbar}{i} \frac{\partial}{\partial_{t} D_{b}^{\beta-1} q} \\
\hat{p}_{0}=\frac{\hbar}{i} \frac{\partial}{\partial t}
\end{gathered}
$$

Note that, 


$$
D_{t}^{0}=1
$$

It is important to notice that if $\alpha$ and $\beta$ are equal unity, the results are found to be inexact agreement with the results that obtained by conventional methods.

\section{Example}

Let us discuss the motion of a pendulum of mass $\mathbf{m}$ and length $l$ with angular displacement $\boldsymbol{\theta}$ from the vertical (Fowles, 1993).

The Lagrangian which describes this example is given by:

$$
L=\frac{1}{2} m l^{2} \dot{\theta}^{2}-m g l(1-\cos \theta)
$$

In the presence of damping the Lagrangian becomes

$$
L=\left[\frac{1}{2} m l^{2} \dot{\theta}^{2}-m g l(1-\cos \theta)\right] e^{\gamma \theta}
$$

For small $\theta$, we have approximately $\cos \theta=1-\frac{\theta^{2}}{2}$

Thus, this Lagrangian reads

$$
L=\left[\frac{1}{2} m l^{2} \dot{\theta}^{2}-\frac{1}{2} m g l \theta^{2}\right] e^{\gamma \theta}
$$

The Lagrangian in fractional form can be written as

$$
L=\left[\frac{1}{2} m l^{2}\left({ }_{0} D_{t}^{\alpha} \theta\right)^{2}-\frac{1}{2} m g l \theta^{2}\right] e^{\gamma \theta}
$$

The Hamiltonian of this system reads

$$
H=\frac{p_{\theta}^{2}}{2 m l^{2}} e^{-\gamma \theta}+e^{\gamma \theta} \frac{m g l}{2} \theta^{2}
$$

Where the fractional canonical momentum is

$$
p_{\theta}=\frac{\partial S}{\partial_{0} D_{t}^{\alpha-1} \theta}
$$

Using equations (30 and 31), equation (14) becomes

$$
H^{\prime}=p_{\circ}+H=\frac{\partial S}{\partial t}+\frac{\left(\partial S / \partial_{0} D_{t}^{\alpha-1} \theta\right)^{2}}{2 m l^{2}} e^{-\gamma \theta}+e^{\gamma \theta} \frac{m g l}{2} \theta^{2}=0
$$

From equation (15), the Hamilton Jacobi function takes the following form

$$
S=S\left({ }_{0} D_{t}^{\alpha-1} \theta, E_{\theta}, t\right)=f(t)+W_{\theta}\left(E_{\theta},{ }_{0} D_{t}^{\alpha-1} \theta\right)+A
$$

Where, $f(t)=-E_{\theta} t$

Inserting equation (33) into equation (32), we obtain

$$
-E_{\theta}+\frac{\left(\partial W_{\theta} / \partial_{0} D_{t}^{\alpha-1} \theta\right)^{2}}{2 m l^{2}} e^{-\gamma \theta}+e^{\gamma \theta} \frac{m g l}{2} \theta^{2}=0
$$

Using separation of variables, we get

$$
\frac{\left(\partial W_{\theta} / \partial_{0} D_{t}^{\alpha-1} \theta\right)^{2}}{2 m l^{2}} e^{-\gamma \theta}=E_{\theta}-\frac{m g l}{2} \theta^{2} e^{\gamma \theta}
$$

Where $E_{\theta}$ is constant.

Solving equation (35), we obtain 


$$
W_{\theta}=\int \sqrt{2 m l^{2}\left(e^{\gamma \theta} E_{\theta}-\frac{m g l}{2} \theta^{2} e^{2 \gamma \theta}\right)} d_{0} D_{t}^{\alpha-1} \theta
$$

Then, the Hamilton Jacobi function is

$$
S=-E_{\theta} t+\int \sqrt{2 m l^{2}\left(e^{\gamma \theta} E_{\theta}-\frac{m g l}{2} \theta^{2} e^{2 \gamma \theta}\right)} d_{0} D_{t}^{\alpha-1} \theta+A
$$

Making use of equation (16), the equation of motion is

$$
\eta_{\theta}=\frac{\partial S}{\partial E_{\theta}}=-t+\int \frac{m l^{2} e^{\gamma \theta}}{\sqrt{2 m l^{2}\left(e^{\gamma \theta} E_{\theta}-\frac{m g l}{2} \theta^{2} e^{2 \gamma \theta}\right)}} d_{0} D_{t}^{\alpha-1} \theta
$$

From equation (31) we get

$$
p_{y}=\frac{\partial S}{\partial_{0} D_{t}^{\alpha-1} y}=\frac{\partial W_{y}}{\partial_{0} D_{t}^{\alpha-1} y}=\sqrt{2 m l^{2}\left(e^{\gamma \theta} E_{\theta}-\frac{m g l}{2} \theta^{2} e^{2 \gamma \theta}\right)}
$$

We are now in a position to quantize our system. The wave function of this example is given by:

$$
\begin{gathered}
\psi\left({ }_{0} D_{t}^{\alpha-1} \theta, t\right)=\frac{1}{\sqrt{p_{\theta}}} \exp \left[\frac{i}{\hbar} S\left({ }_{0} D_{t}^{\alpha-1} \theta, E_{\theta}, t\right)\right] \\
\psi=\left[2 m l^{2}\left(e^{\gamma \theta} E_{\theta}-\frac{m g l}{2} \theta^{2} e^{2 \gamma \theta}\right)\right]^{\frac{-1}{4}} \exp \left\{\frac{i}{\hbar}\left[-E_{\theta} t+\int \sqrt{2 m l^{2}\left(e^{\gamma \theta} E_{\theta}-\frac{m g l}{2} \theta^{2} e^{2 \gamma \theta}\right)} d_{0} D_{t}^{\alpha-1} \theta+A\right]\right\}
\end{gathered}
$$

The Schrödinger equation takes the form

$$
\hat{H}^{\prime} \psi=\left\{\frac{\hbar}{i} \frac{\partial}{\partial t}-e^{-\gamma \theta} \frac{\hbar^{2}}{2 m l^{2}} \frac{\partial^{2}}{\partial\left({ }_{0} D_{t}^{\alpha-1} \theta\right)^{2}}+\frac{m g l}{2} \theta^{2} e^{\gamma \theta}\right\} \psi=0
$$

After some calculations, it is easy to show that in the semiclassical limit $h \rightarrow 0, \hat{H} \psi=E \psi$

\section{Conclusion}

The damped systems are investigated using the Hamilton Jacobi quantization scheme. The fractional Hamilton-Jacobi function $\mathbf{S}$ is determined using the method of separation of variables in the same manner as for regular systems. The equations of motion were derived from this function. Further, this function enables us to formulate the wave function; this meant that the quantization using the fractional WKB approximation had been completed. The solution of the illustrative example is found to be in exact agreement with the usual classical mechanics for regular Lagrangian when $\alpha, \beta$ are equal unity only. Also in the semiclassical limit $\hbar \rightarrow 0$, the quantum results are found to be in exact agreement with the classical results.

\section{References}

Arnold, V. I. (1989). Mathematical Methods of Classical Mechanics (4th ed). Berlin: Springer-Verlag.

Fowles. G. (1993). Analytical Mechanics (5th ed). Harcourt Brace andCompany, Florida.

Goldstein, H. (1980). Classical Mechanics (2nd ed.). Addison-Wesley, Reading-Massachusetts.

Guler, Y. (1992). Integration of Singular Systems. Il Nuovo Cimento B, 107(12), 1389-1395. Retrieved from https://link.springer.com/article/10.1007/BF02722849

Hasan, E. H. (2016). Fractional Quantization of Holonomic Constrained Systems Using Fractional WKB Approximation. Advanced Studies in Theoretical Physics, 10(5), 223-234. http://dx.doi.org/10.12988/ astp.2016.6313

Hasan, E. H., Rabei, E. M., \& Ghassib, H. B. (2004). Quantization of Higher-Order Constrained Lagrangian Systems Using the WKB Approximation. International Journal of Theoretical Physics, 43(4), 1073-1096. Retrieved from http://link.springer.com/article/10.1023/B:IJTP.0000049027.45011.37 
Jarab'ah, O., Nawafleh, K., \& Ghassib, H. (2013). Canonical Quantization of Dissipative Systems. European Scientific Journal, 9(36), 132-154. Retrieved fromhttp://www.eujournal.org/index.php/esj/article/download/ 1946/1888

Lanczos. C. (1986). The Variational Principles of Mechanics. Dovers Publications.

Muslih, S. I. (2002). Quantization of Singular Systems with Second-Order Lagrangians. Modern Physics Letters A, 17(36), 2383-2391.

Nawafleh, K. I. (1998). Constrained Hamiltonian Systems: A Preliminary Study. M.Sc. Thesis, University of Jordan, Amman, Jordan.

Nawafleh, K., Rabei, E., \& Ghassib, H. (2004). Hamilton-Jacobi Treatment of Constrained Systems, International Journal of Modern Physics A, 19(3), 347-354. https://doi.org/10.1142/s0217751x04017719

Nawafleh, K. (2007). Hamilton-Jacobi Formulation with Gauge Conditions. Dirasat, Pure Sciences, 34(1), 119-124. https://journals.ju.edu.jo/DirasatSci/article/view/431

Rabei, E., \& Guler, Y. (1992). Hamilton - Jacobi Treatment of Second-Class Constraints. Physical Review A, 46(6), 3513-3515. Retrieved from http://link.aps.org/pdf/101103/physRevA.46.3513

Rabei, E. M. (1999). Canonical Treatment of Regular Lagrangians with Holonomic Constraints as Singular Systems. Turkish Journal of Physics, 23, 1083-1091. http://adsabs.harvard.edu/abs/2000NCimB.115.1159R

Rabei, E. M., Nawafleh, K. I., and Ghassib, H. B. (2002). Quantization of Constrained Systems Using the WKB Approximation. Physical Review A, 66, 024101-024106.

Rabei, E. M., Hasan, E. H., and Ghassib, H. B. (2005). Quantization of Second-Order constrained Lagrangian Systems Using the WKB Approximation. International Journal of Geometric Methods in Modern Physics, 2(3), 458-504.

Rabei, E.M., Altarazi, I.M.A. Muslih, S.I. and D. Baleanu. (2009). Fractional WKB Approximation. Nonlinear Dynamics, 57, 171-175. http://dx.doi.org/10.1007/s11071-008-9430-7

Rabei, E.M. Muslih, S.I. and D. Baleanu (2010). Quantization of fractional systems using WKB approximation. Communication in Nonlinear Science and Numerical Simulation, 15(4), 807-811. http://dx.doi.org/10.1016/ j.cnsns.2009.05.022

\section{Copyrights}

Copyright for this article is retained by the author(s), with first publication rights granted to the journal.

This is an open-access article distributed under the terms and conditions of the Creative Commons Attribution license (http://creativecommons.org/licenses/by/4.0/). 